\title{
La respuesta educativa a los alumnos más capaces en los planes de atención a la diversidad
}

\author{
Lorenzo Pérez Diez a \\ Carmen Jiménez Fernández ${ }^{b}$
}

\section{Resumen}

La atención a la diversidad es un principio básico del sistema educativo español y su finalidad es dar respuesta a las necesidades de los estudiantes escolarizados en sus centros. En esta diversidad, los estudiantes de altas capacidades han ido adquiriendo cierta visibilidad en el diagnóstico y la intervención. La normativa nacional establece el Plan de Atención a la Diversidad (PAD de aquí en adelante) como el instrumento básico para planificar, implementar y evaluar las medidas de atención a la diversidad de diferentes colectivos, entre ellos, el de los alumnos más capaces. Mediante la técnica del análisis de contenido, esta colaboración analiza los PAD en una muestra de centros escolares de la provincia de Palencia perteneciente a la Comunidad Autónoma de Castilla y León. Concluye que, en dichos planes, la presencia de medidas educativas dirigidas a los más capaces es nula o meramente testimonial no trascendiendo más allá de la normativa en la que se fundamentan, resultando ser una necesidad desarrollar estos planes en la práctica educativa real.

Palabras clave: Altas capacidades. Identificación. Intervención educativa. Plan de atención a la diversidad.

\section{Introducción}

Cada vez es más difícil ignorar la amplitud de las diferencias que se producen en los centros educativos y en sus las aulas y, por ello, la atención a la diversidad es un principio básico en el que se fundamentan las políticas educativas en los distintos países. En España dicho principio está recogido en las leyes generales que regulan la Educación a nivel nacional, así como en las de ámbito autonómico.

\footnotetext{
a Universidad Nacional de Educación a Distancia, Escuela Internacional de Doctorado, Madrid, España.

b Universidad Nacional de Educación a Distancia, Facultad de Educación, Madrid, España.
} 
En el caso de los estudiantes con altas capacidades, el principio de atención a la diversidad ha significado que sean más visibles en el sistema escolar, no solo porque aparecen de forma explícita en las leyes generales de la Educación (ESPAÑA, 2006; 2013; 2016), sino también porque se han incluido en la normativa una serie de medidas que pretenden incidir en su realidad educativa. Sin embargo, la práctica educativa presenta déficits y carencias que repercuten en la calidad de la Educación que estos alumnos reciben.

Por un lado, los desarrollos normativos no han venido acompañados de iniciativas que impulsen la intervención con estos alumnos en los centros educativos (JIMÉNEZ et al., 2010) y, por otro, la ausencia de una definición relativamente unívoca de alta capacidad en la legislación educativa produce desajustes entre los procedimientos de identificación y la posterior intervención que de ella se deriva (HERNÁNDEZ TORRANO; GUTIÉRREZ SÁNCHEZ, 2014).

Los bajos porcentajes de identificación del alumnado de altas capacidades en el sistema educativo (Gráfico) respecto a las estimaciones de autores e instituciones indicadas en la Tabla 1 tienen como consecuencia que un elevado número de estos alumnos no tengan respuesta a sus necesidades educativas al no ser identificados como tales.

Gráfico - Porcentajes de alumnos de altas capacidades identificados en el sistema educativo español

Promedios de identificación de alumnos de altas capacidades en Educación Primaria y Secundaria

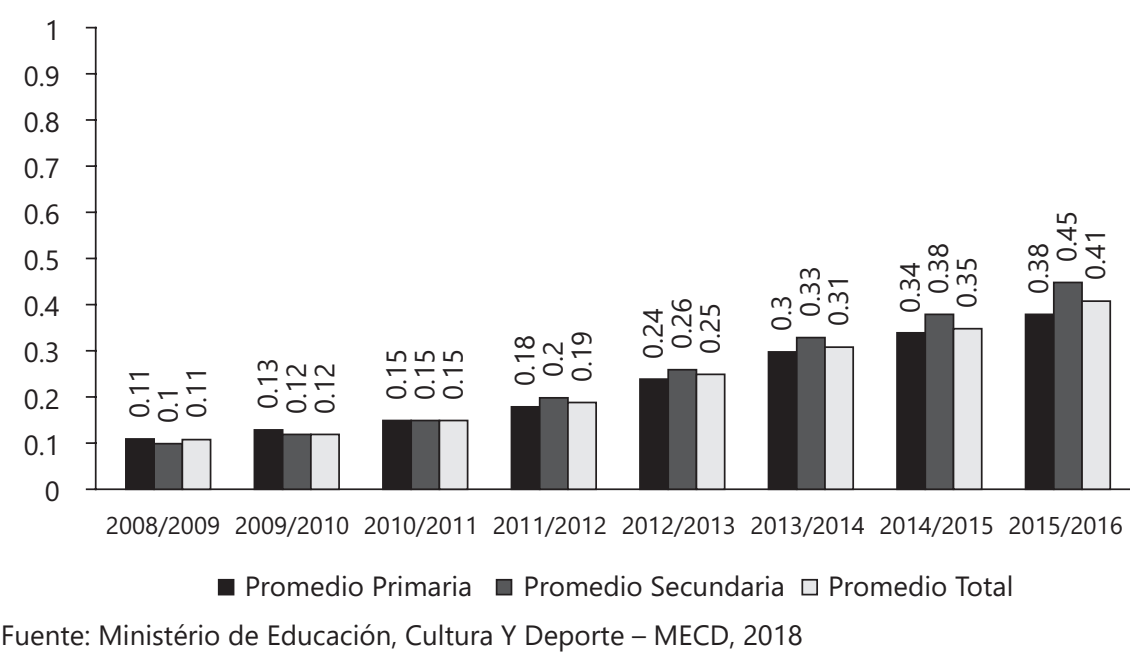


Tabla 1 - Estimaciones de autores e instituciones sobre la prevalencia de la alta capacidad

\begin{tabular}{lc}
\hline Autores e Instituciones & Porcentajes \\
\hline Comité Económico y Social Europeo (2013) & $5 \%-10 \%$ \\
Renzulli (2005) & $20 \%$ \\
Gagné (2015) & $10 \%$ \\
\hline
\end{tabular}

Fuente: Elaboración propia (2018)

Los estudios sobre altas capacidades muestran los factores que inciden en su Educación y la complejidad derivada de su interrelación. Y una de las conclusiones es que la "congruencia entre el sistema de identificación y la intervención posterior es tan importante, que incluso podría ser considerada la "regla de oro" de la Educación del alumnado con alta capacidad intelectual" (RENZULLI; GAESSER, 2015, p. 104).

Ante esta situación, es necesario reflexionar sobre los factores que influyen en la manera en que se está trasladando a la práctica educativa el principio de atención a la diversidad en la Educación de los alumnos de altas capacidades.

Las páginas que siguen se centran en el análisis del contenido de las medidas implícitas y explícitas que contienen los Plan de Atención a la Diversidad (PAD) y su relación con la calidad de la Educación que reciben los más capaces y, más concretamente, si con dichas medidas están en disposición de obtener los mejores resultados individuales y sociales. Procederemos del modo siguiente. En primer lugar, se resumen las ideas contenidas en la normativa educativa que regula la alta capacidad. A continuación, se esboza la organización escolar en la que se produce la atención a los más capaces. Para finalizar, se expondrá el estudio empírico propiamente dicho o aplicación de la técnica del análisis de contenido a los PAD de los centros de Educación primaria seleccionados para este estudio. La finalidad del análisis de contenido es examinar y evaluar de forma crítica la situación educativa de los alumnos de altas capacidades en los PAD elaborados por los centros seleccionados, siguiendo unas reglas previamente determinadas.

\section{Normativa y atención a la diversidad}

La normativa es un factor de gran interés en la Educación de los alumnos de altas capacidades (HERNÁNDEZ TORRANO; GUTIÉRREZ SÁNCHEZ, 2014), pues regula su nivel de visibilidad en el sistema educativo, denominaciones 
utilizadas, conceptualización subyacente, procedimientos de identificación y proceso de intervención.

En este ámbito, el principio clave es la atención a la diversidad y aunque otros factores como la autonomía organizativa y pedagógica de cada centro permiten diseñar e implementar una respuesta educativa adaptada al alumnado de altas capacidades, dicho principio educativo es el núcleo central de las propuestas realizadas y se conceptualiza como un conjunto de acciones educativas para prevenir y responder a las necesidades temporales o permanentes de todos los estudiantes, así como a sus diferencias.

En la legislación nacional dichas acciones se explicitan con carácter general en medidas ordinarias y extraordinarias. En el caso de los más capaces, se concretan en la flexibilización educativa que puede tomar dos formas: anticipación de un curso en el inicio de la escolarización o reducción de la misma teniendo en cuenta las posibilidades de socialización del alumno en el nuevo grupo. Otras medidas son la organización en grupos flexibles, el cambio de dinámicas de trabajo, el favorecimiento de las relaciones de comunicación entre el alumnado y la organización flexible de espacios y tiempos.

En el caso de la Comunidad Autónoma de Castilla y León, dichas medidas se recogen en el Plan de Atención a la Diversidad de cada centro educativo. Estos planes, al estar contemplados en la normativa, contienen una serie de elementos comunes que se pueden diferenciar en la práctica educativa según cada entorno escolar y según las características del propio centro. Dichos elementos se recogen simplificados en la Figura 1 (ESPAÑA, 2016):

\section{Organización educativa y atención a los alumnos de altas capacidades}

Los centros que forman parte del sistema educativo asientan su práctica en una estructura organizativa conformada por una red de relaciones en la que interactúan los miembros de la comunidad educativa y que, a su vez, reciben influencias del entorno (CARDONA ANDÚJAR, 2008).

Esta estructura organizativa es un elemento importante para la práctica educativa. Por ejemplo, si los espacios o la distribución del tiempo no son adecuados (GAIRÍN; RODRÍGUEZ GÓMEZ, 2011) o si no propician una toma de decisiones que mejore el aprendizaje de los alumnos de altas capacidades, entonces los cambios organizativos necesarios no se producirán (GAIRÍN; GOIKOETXEA, 2008) y repercutirán en la calidad de la Educación que estos alumnos reciben. 
Figura 1 - Elementos del plan de atención a la diversidad

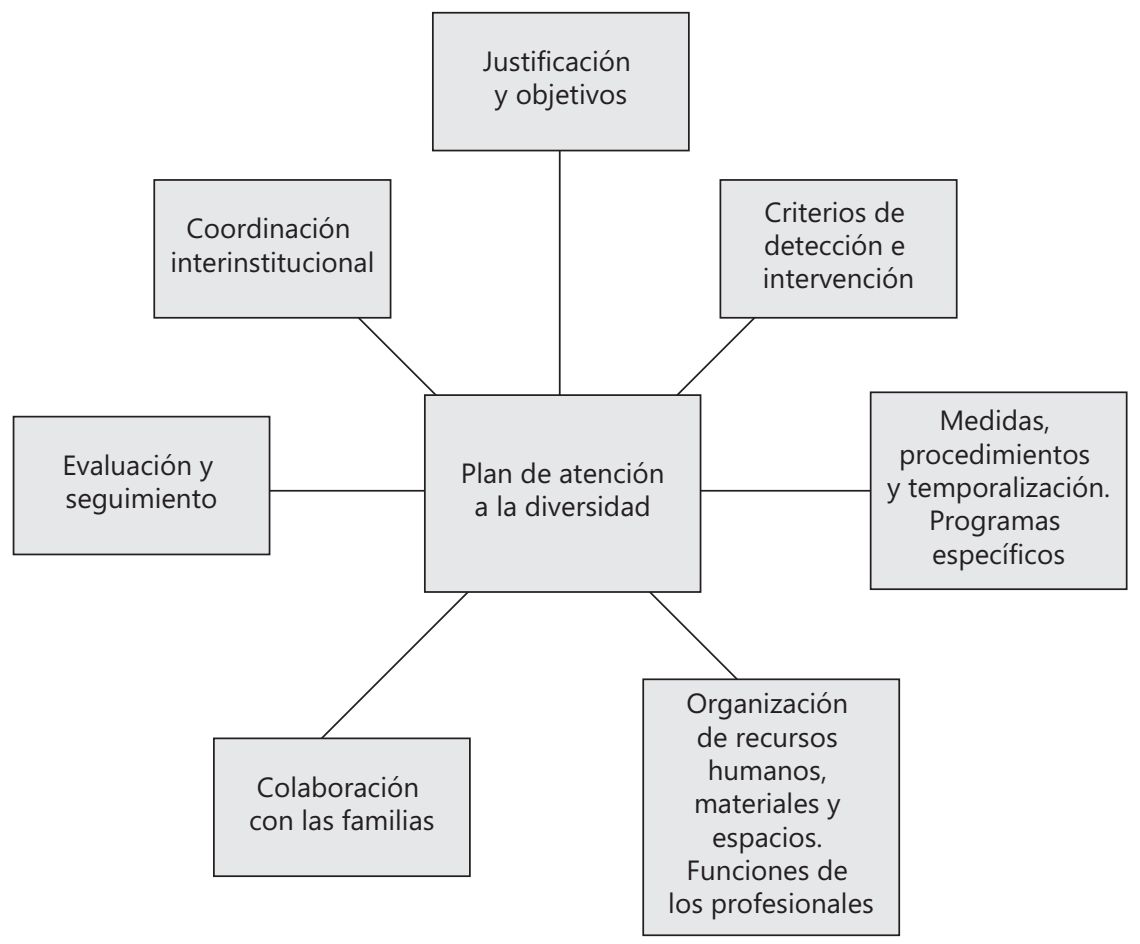

Fuente: España, 2016

En el ámbito organizativo, se abordan los siguientes componentes: agrupamiento del alumnado, organización del profesorado, espacios y tiempos, que sirven de referente en el análisis de contenido de las actuaciones y medidas organizativas y educativas que inciden en la Educación de los alumnos de altas capacidades y que están recogidas en los planes de atención a la diversidad anteriormente citados.

Agrupamiento del alumnado. Tomando como referencia el principio de atención a la diversidad, todos los alumnos tienen derecho a un ambiente educativo flexible que esté en consonancia con su ritmo de aprendizaje.

Las diferentes formas de agrupamiento, ya sea con base a niveles educativos o grupos de Enseñanza, inciden de forma diferente en las posibilidades de intervención con los más capaces (Figura 2). 
Figura 2 - Agrupamiento del alumnado de altas capacidades en función de los niveles educativos

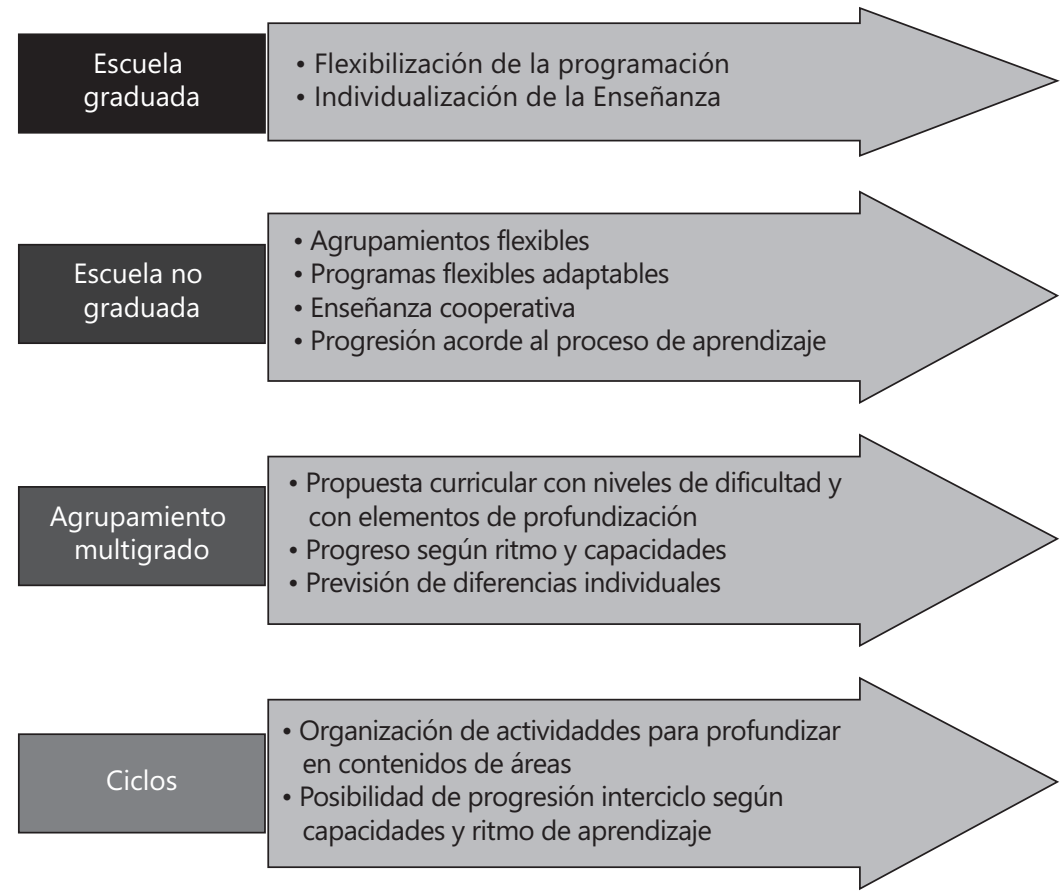

Fuente: Elaboración propia (2018)

En lo que respecta a los grupos de Enseñanza, la cuestión principal está en esclarecer si los alumnos de altas capacidades aprenden mejor en grupos homogéneos, es decir, con similares niveles de capacidad, o heterogéneos, esto es, con una amplia gama de habilidades.

Investigaciones como la de Walker, Shore y French (2011) concluyen que los agrupamientos homogéneos benefician especialmente a los alumnos de altas capacidades al presentar un mejor rendimiento y la posibilidad de intervenir con un único tipo de necesidades educativas.

El estudio de metaanálisis de Steenbergen-Hu, Makel y Olszewski-Kubilius (2016) concluyó que los efectos de agrupar a los más capaces de forma homogénea fueron positivos, moderados y estadísticamente significativos. Estos estudiantes se vieron beneficiados en su aprendizaje al ubicarse en grupos especiales o en programas diseñados específicamente para ellos. 
En este mismo estudio de metaanálisis, también se encontraron investigaciones que concluían que los agrupamientos por capacidad en las escuelas no producen mejoras académicas para los estudiantes de altas capacidades y obstaculizan el aprendizaje de los estudiantes normales.

Por otro lado, al relacionar el agrupamiento por capacidad con la equidad educativa, el resultado es que los estudiantes que pertenecen a minorías están subrepresentados en los programas para dotados y talentosos. Desde un punto de vista general, el sistema de agrupar estudiantes por capacidad tiene como consecuencia que las escuelas estructuren la desigualdad (OAKES, 1985).

Desde otra perspectiva, las investigaciones sobre grupos heterogéneos concluyen que se produce un rendimiento escolar bajo en los alumnos de altas capacidades y tienden a limitar su progreso al plantear tareas demasiado fáciles que disminuyen su motivación (WALKER; SHORE; FRENCH, 2011).

La decisión sobre cómo agrupar a los más capaces no puede ser una cuestión de todo o nada. Winebrenner y Brulles (2008) plantean la necesidad de que los alumnos más capaces se relacionen con sus iguales en edad para socializarse y adquirir competencias básicas del diario aprendizaje, y con sus iguales en capacidad para desarrollar su potencial mediante desafíos adecuados a sus recursos cognitivos.

Organización del profesorado. Se plantea desde una doble perspectiva: relaciones orgánicas y educativas. La Figura 3 sintetiza las posibles influencias de estas relaciones en la Educación de los alumnos de altas capacidades.

Tanto los elementos de nivel orgánico como las relaciones que se producen entre ellos, además de las diferentes formas de trabajo en equipo del profesorado, permiten dar una respuesta educativa a los alumnos de altas capacidades al articularse distintos tipos de actuaciones en las que se potencia la participación y la investigación para la práctica educativa.

Espacios y tiempos. Aceptando que la determinación del espacio debe ser posterior a la propuesta de actividades realizada, un espacio homogéneo propiciará una intervención educativa homogénea; por tanto, debería configurarse desde una perspectiva flexible en la que lo fundamental sea la actividad y la participación del alumno.

Respecto a los alumnos más capaces, la reflexión acerca de cómo influye el espacio en su actividad permitirá realizar las modificaciones adecuadas para un proceso que dé respuesta a sus necesidades específicas. 
Figura 3 - Relaciones orgánicas y educativas y su incidencia en la Educación de los más capaces
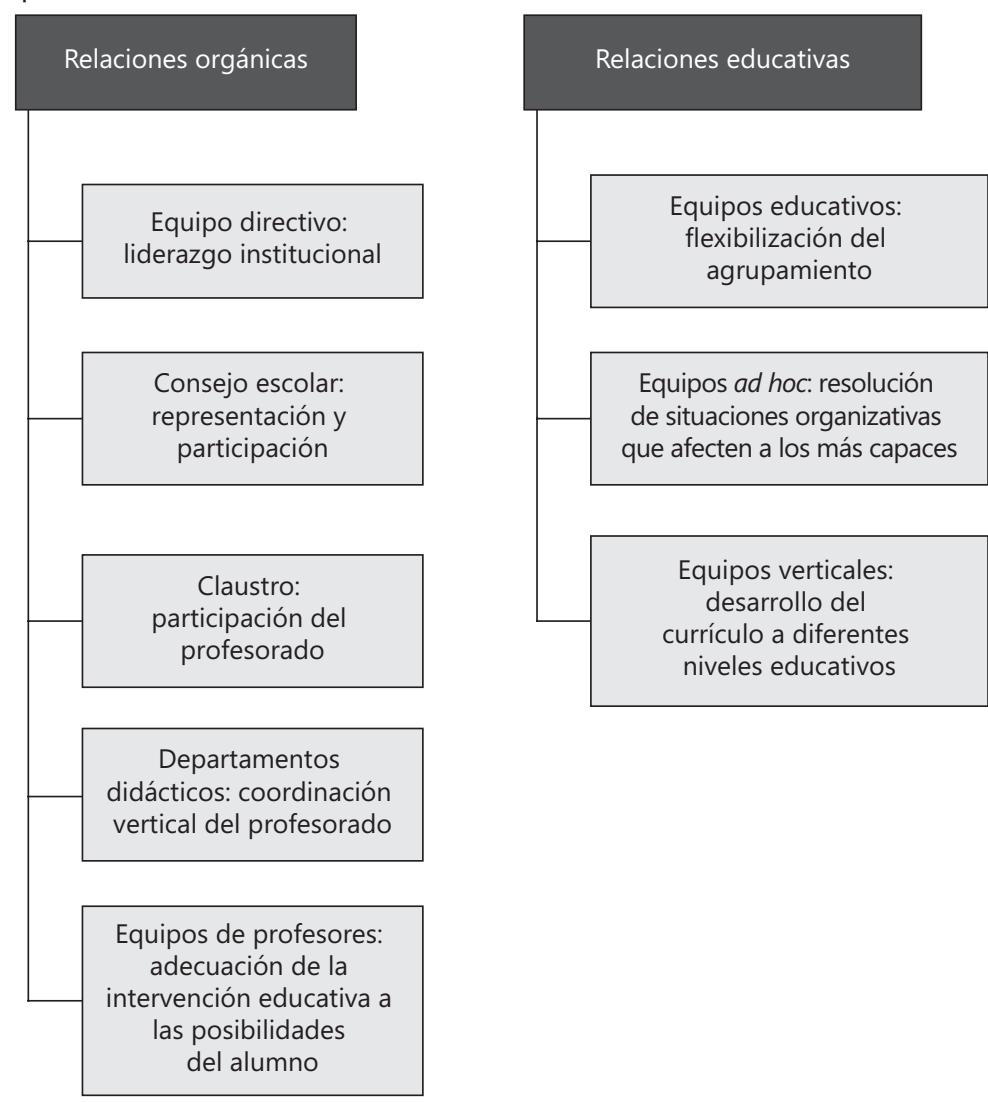

Fuente: Elaboración propia (2018)

En cuanto al tiempo, decir que es un componente de la estructura organizativa que influye en la construcción de la red de relaciones de dicha estructura y que condiciona las que se producen en ella. Tanto para los alumnos como para el profesorado el tiempo es limitado y es necesario priorizarlo para abordar lo importante. La organización del tiempo para los alumnos de altas capacidades debería tener en cuenta lo siguiente:

- El ritmo de trabajo estable propiciado por la distribución tradicional del tiempo no beneficia su ritmo de trabajo más rápido. Es necesario que exista flexibilidad en la construcción de los horarios y proporcionar alternativas a los programas de trabajo para que no sean uniformes durante todo el curso. 
- Esta estabilidad temporal favorece que los alumnos terminen adaptándose, desaprovechándose su ritmo de trabajo. Hay que posibilitar que los alumnos de altas capacidades adquieran la capacidad para organizar su propio tiempo.

- La vinculación a una red de horarios tiene como consecuencia que las materias y las actividades se distribuyan de forma fragmentada. Debe poder alterarse la duración de las unidades de trabajo y su localización en el horario; para ello, dicho horario tiene que estar supeditado a la propuesta didáctica que se quiera desarrollar.

Para superar estas limitaciones, una opción a tener en cuenta sería el aprendizaje personalizado, que fomenta un entorno centrado en el estudiante y que le anima a ser responsable de sus trayectorias de aprendizaje. En un ambiente con estas características, los alumnos de altas capacidades tendrían la posibilidad de determinar la estrategia y el ritmo al que aprenden (INSTITUTO NACIONAL DE TECNOLOGÍAS EDUCATIVAS Y DE FORMACIÓN DEL PROFESORADO (INTEF), 2014; 2015; 2016).

Para hacer efectiva la personalización del aprendizaje, las tecnologías de aprendizaje adaptativo (INTEF, 2015) permitirían readaptar las necesidades de aprendizaje de cada individuo mediante software y plataformas en línea. El aprendizaje en línea (INTEF, 2016) se encargaría de proporcionar a los estudiantes oportunidades educativas formales e informales a través de Internet.

Trabajo en red y nuevas tecnologías de la información y la comunicación. Las tecnologías de la información y la comunicación (TIC, en adelante) influyen fuertemente en la práctica educativa en todos los niveles; de ahí que su influencia también se manifieste en los elementos de la estructura organizativa antes mencionados: agrupamiento del alumnado, organización del profesorado, espacios y tiempos. En el informe de tendencias TIC Kennisnet del INTEF (2017, p. 22), se indica que:

Los niños están acostumbrados a usar tabletas en el hogar para realizar toda clase de acciones en Internet, como aprender a leer, escribir o hacer más, recurriendo a aplicaciones educativas. Sin embargo, los centros escolares a menudo siguen anclados en sus antiguos métodos: los niños buscan información en libros y escriben sus tareas en cuadernos de actividades, que son calificadas a mano.

La normativa educativa plantea la necesidad de adecuar el proceso educativo a las características del sujeto que aprende. La desubicación espacial y la flexibilización 
de los tiempos de Enseñanza y aprendizaje son una consecuencia de la irrupción de las TIC en el proceso educativo.

Los modelos de aprendizaje híbrido (INTEF, 2014; 2015) que combinan trabajo presencial en el aula y el aprendizaje en línea o el rediseño de los espacios de aprendizaje (INTEF, 2016) que implica que las aulas estén preparadas para acoger y acomodar diversos tipos de dispositivos y permitir la conectividad a Internet para promover la interactividad, son ejemplos que desafían las estructuras organizativas tradicionales y proporcionan propuestas alternativas para educar a los más capaces.

Tourón y Tourón (2008) han señalado las posibilidades y ventajas de las metodologías de Enseñanza y aprendizaje a distancia o "e-learning" con el soporte de las tecnologías de la información y la comunicación (TICs) para atender las necesidades educativas específicas de alta capacidad.

El "e-learning" permite disponer de un tutor a distancia con el cual desarrollar planes de aprendizaje altamente individualizados; interactuar con alumnos de otros centros, creando comunidades virtuales de aprendizaje; individualizar el ritmo y el contenido de aprendizaje tanto en amplitud como en profundidad.

Otro elemento a tener en cuenta son las plataformas educativas "on-line" $\mathrm{y}$, entre ellas, se puede destacar la Khan Academy (ROMÁN GONZÁLEZ, 2014) cuyas características y principios pedagógicos le permiten converger de forma adecuada con el aprendizaje que despliegan los alumnos de altas capacidades: posibilidad de relacionarse con iguales en capacidad, avance al propio ritmo e incluso asesoramiento ocasional por parte de tutores.

También el modelo de "flipped classroom" permite que los alumnos más capaces sean protagonistas de su aprendizaje pues se respeta el ritmo y profundidad de lo que aprenden y esto les permite avanzar a la velocidad acorde con su capacidad (TOURÓN; SANTIAGO, 2015).

\section{Estudio de campo: el análisis de contenido y sus resultados}

Como se ha indicado, los PAD son los documentos que incluyen el diseño y la planificación de medidas organizativas y actuaciones educativas para ser implementadas en los centros y adecuar su intervención a las necesidades de los alumnos en ellos escolarizados. Las fases seguidas en la realización del análisis de contenido y los resultados obtenidos han sido las siguientes. 


\subsection{Población y muestra}

La población incluye un total de 53 centros públicos de Educación primaria de la provincia de Palencia. La muestra está conformada por cinco centros de dicha población (Figura 4), elegidos de modo que estuvieran representados los distintos tipos de centros de la provincia.

El análisis de contenido se ha realizado en dos fases:

- Primera fase. El análisis se realizó en los centros educativos de las localidades de Astudillo y Carrión de los Condes, dos en total.

- Segunda fase. Los tres centros seleccionados pertenecen a las localidades de Osorno, Becerril de Campos y Villamuriel de Cerrato.

Figura 4 - Centros de la provincia de Palencia seleccionados en primera y segunda fase para el análisis de contenido (2019)

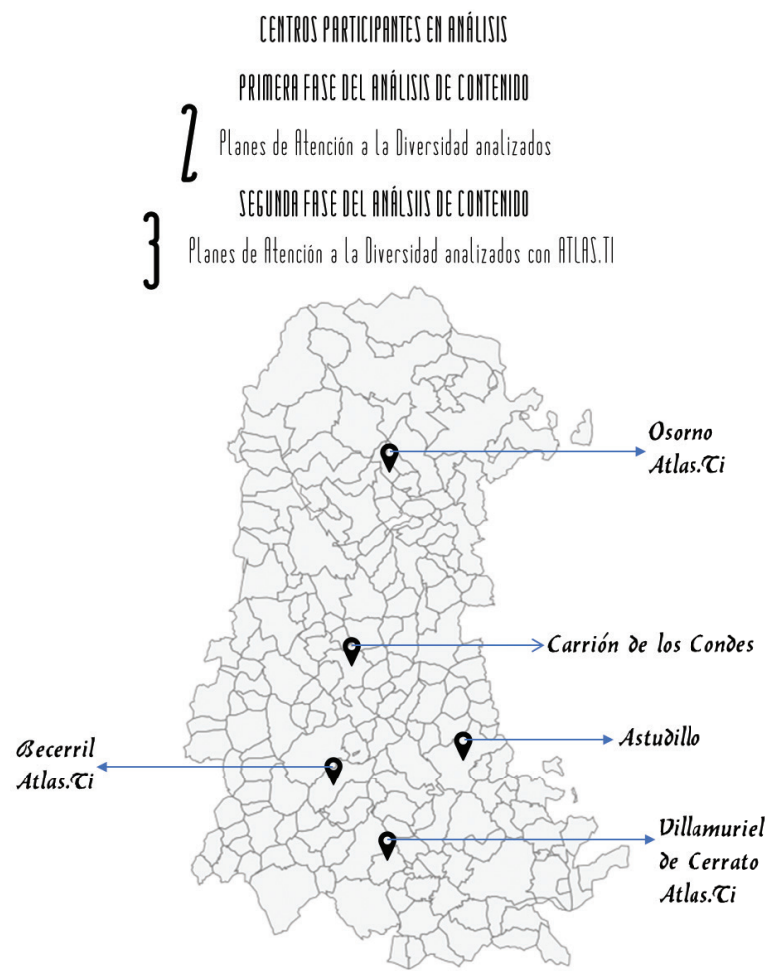

Fuente: Elaboración propia (2018) 


\subsection{El análisis de contenido como instrumento de recogida de información}

La comunicación lingüística tiene gran importancia en el ámbito educativo ya que suministra cantidad de datos en las investigaciones relacionadas con la Educación y las ciencias sociales en general (GARCÍA; GONZÁLEZ; BALLESTEROS, 2001). El análisis de contenido es un conjunto de técnicas mediante las que se analizan las comunicaciones lingüísticas escritas que permiten describir, en nuestro caso, el contenido de los mensajes que aparecen en los PAD de los cinco centros seleccionados para la investigación.

Según se infiere de la normativa, dichos planes deben contener información acerca de la intervención con los más capaces y, mediante su análisis, se podrán obtener conclusiones que relacionen la estructura organizativa de los centros con la intervención que se hace con los más capaces.

Tomando como referencia los PAD, la intervención con los más capaces debe tener las siguientes características:

- Estos alumnos tienen que estar escolarizados en centros ordinarios;

- Los centros pueden desarrollar planes de actuación específicos adecuados a sus necesidades educativas;

- Se pueden realizar agrupamientos de los alumnos más capaces con alumnos de cursos superiores para el desarrollo de una o varias áreas del currículo;

- Se pueden aplicar medidas de enriquecimiento curricular en el aula ordinaria

- Se pueden utilizar modelos organizativos flexibles;

- Se puede realizar la flexibilización excepcional del periodo de escolarización siempre que las medidas anteriores sean insuficientes.

Para conocer cómo es la intervención con los alumnos de altas capacidades en los PAD, lo conveniente y congruente sería que algunas o todas estas medidas estén contempladas en dichos planes y así conocer cuál es su situación en la propuesta educativa que realizan los centros. 
En cada una de las dos muestras de centros el análisis de contenido ha seguido un procedimiento parcialmente distinto.

Primera fase. Se seleccionaron los dos centros señalados y el análisis de contenido fue realizado por el propio investigador siguiendo el procedimiento clásico siguiente:

a) Selección de las frases y párrafos importantes. Constituyen los fragmentos significativos de cada plan;

b) Unidades de análisis. Las frases y párrafos seleccionados se han analizado como núcleos de significado de la comunicación al proporcionar información sobre los PAD;

c) Reglas de enumeración. Indican las interacciones educativas entre los actores del proceso educativo y los alumnos de altas capacidades;

d) Categorías. Constituidas por elementos comunes contenidos en las unidades de análisis. Se caracterizan por ser excluyentes, amplias y exhaustivas, definidas por el mismo principio, claras y consistentes, replicables y productivas.

Segunda fase. Se realizó en los tres centros indicados y contó, además, con la utilización de la herramienta informática ATLAS.TI que sirvió para agilizar la selección, reducción e interpretación de los datos.

La utilización de ATLAS.TI presenta las siguientes características procedimentales:

a) Citaciones. O selección de los fragmentos importantes de los planes de atención analizados;

b) Codificación. Implica segmentar los datos según el objetivo planteado y sintetizarlos en unidades analizables para ser interpretados. Las unidades obtenidas fueron las siguientes:

a) Atención a la diversidad;

b) Atención educativa no ordinaria;

c) Atención educativa ordinaria;

d) Intervención educativa temprana;

e) Respuesta educativa inclusiva; 
c) Creación de memos a partir de la reflexión sobre la investigación y mediante inferencias analíticas a partir de los datos;

d) Familias. Agrupaciones de los elementos anteriores de acuerdo a su similitud con posibles categorías.

La Tabla 2 sintetiza el procedimiento seguido con las denominaciones propias de cada fase según la forma de análisis.

Tabla 2 - Procedimiento seguido en el análisis de contenido en cada una de las fases (2018)

\begin{tabular}{ccc}
\hline & Primera etapa: Investigador & Segunda etapa: ATLAS.TI \\
\cline { 2 - 3 } & Planes de atención a la diversidad & Documentos primarios \\
\hline $1^{\circ}$ & Selección de frases y párrafos importantes & Citaciones \\
$2^{\circ}$ & Unidades de análisis & Codificación \\
$3^{\circ}$ & Reglas de enumeración & Memos \\
$4^{\circ}$ & Categorías & Familias \\
\hline
\end{tabular}

Fuente: Elaboración propia (2018)

\subsection{Resultados obtenidos}

En el procedimiento seguido para realizar el análisis de contenido es interesante destacar la importancia de las reglas de enumeración en la primera fase y de los correspondientes memos en la segunda (Figura 5). Su valor está en que ponen de manifiesto las interacciones educativas, en el primer caso, y la realización de inferencias a partir de los datos, en el segundo.

En la segunda fase, la regla de enumeración relacionada con el orden desapareció ya que estaban secuenciados por la normativa educativa; de ahí que no indicasen orden o prioridad en la intervención con los más capaces. Por otro lado, la dualidad implícito/explícito tiene el siguiente significado:

- Explícito: términos que indican de forma expresa que se trata de elementos vinculados a las altas capacidades o a otros alumnos con necesidades educativas

- Implícito: términos que indican un relación más difusa y no directa con las altas capacidades 
Figura 5 - Reglas de enumeración y evolución en memos para el análisis mediante ATLAS.TI (2016)

\begin{tabular}{|l|}
\hline \multicolumn{1}{|c|}{ Reglas de enumeración } \\
\hline Presencia /ausencia de contenidos implícitos/explícitos vinculados a las altas capacidades \\
$\begin{array}{l}\text { Frecuencia de los contenidos explícitos/implícitos vinculados a las altas capacidades o } \\
\text { términos vinculados a las mismas } \\
\text { Frecuencia ponderada de los contenidos explícitos/implícitos vinculados a las mismas } \\
\text { Orden o secuencia de aparición de los elementos explícitos/implícitos vinculados a las } \\
\text { altas capacidades }\end{array}$ \\
\hline \multicolumn{1}{c|}{ Memos } \\
\hline Referencia implícita a los alumnos de altas capacidades \\
Referencia explícita a los alumnos de altas capacidades \\
Frecuencia de contenidos implícitos vinculados a las altas capacidades \\
Frecuencia de contenidos explícitos vinculados a las altas capacidades \\
Frecuencia de contenidos explícitos vinculados a otros alumnos con necesidades educativas \\
\hline
\end{tabular}

Fuente: Elaboración propia (2018)

En los PAD de la primera fase, respecto a las reglas de enumeración, los resultados fueron los siguientes (Figuras 6 y 7 ).

Figura 6 - Resultados del análisis de las reglas de enumeración del PAD de Astudillo (2016)

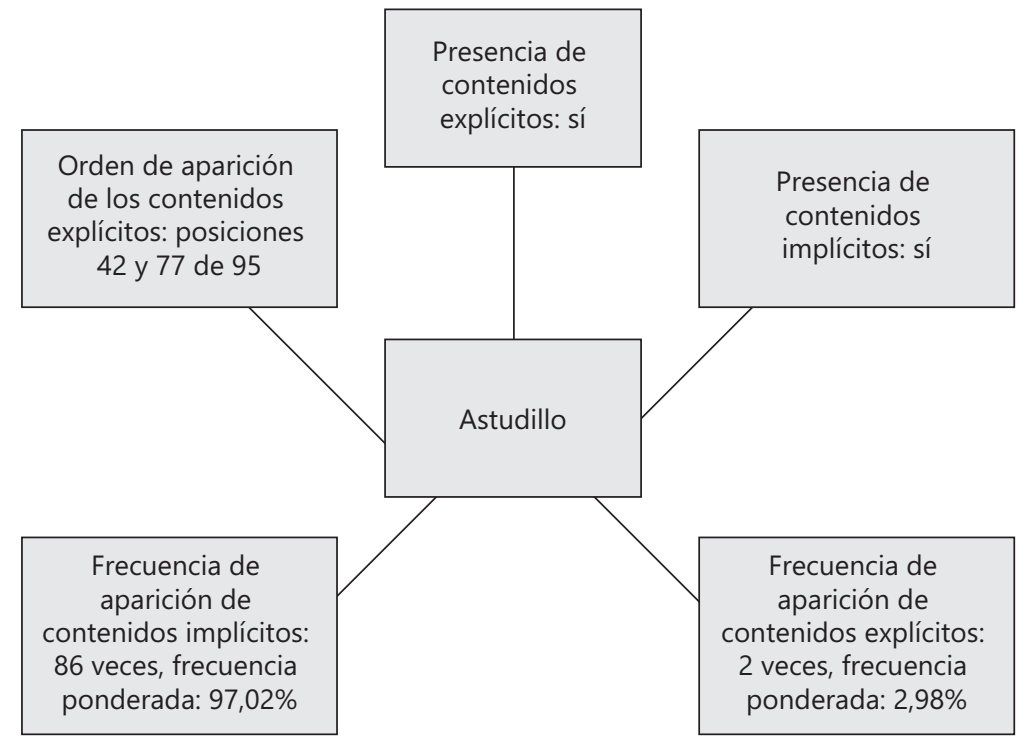

Fuente: Elaboración propia (2018) 
Figura 7 - Resultados del análisis de las reglas de enumeración del PAD de Carrión de los Condes (2016)

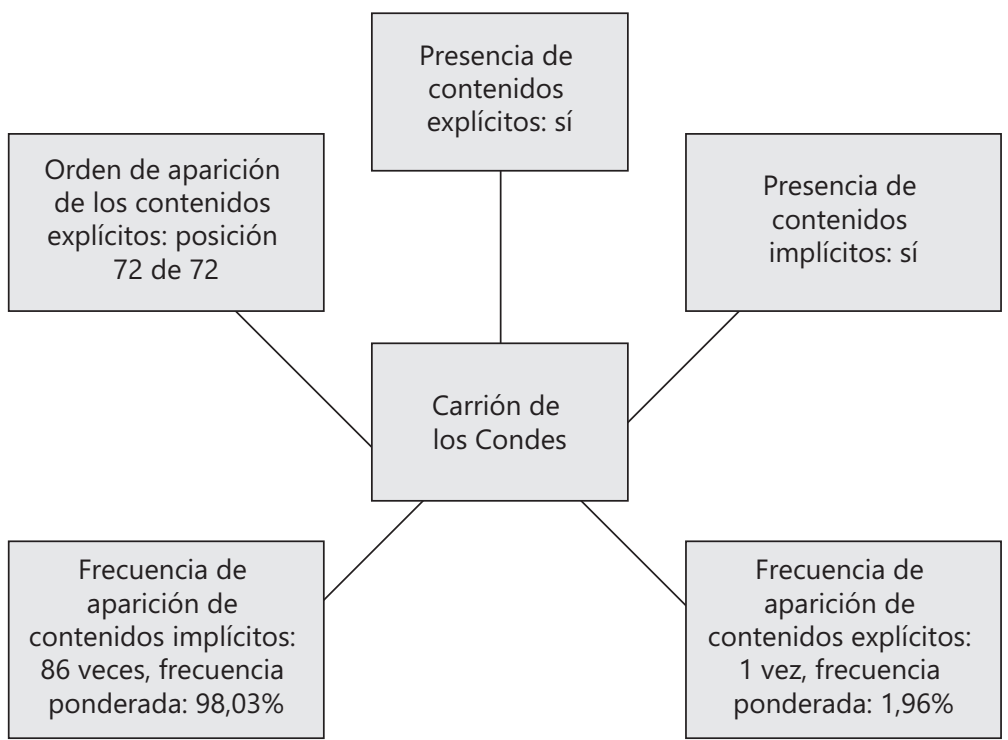

Fuente: Elaboración propia (2018)

Respecto a los de la segunda fase, la Tabla 3 resume los resultados.

Tabla 3 - Reglas de enumeración de la segunda fase del análisis de contenido (2018)

\begin{tabular}{lccc}
\hline Reglas de enumeración & Osorno & $\begin{array}{c}\text { Becerril de } \\
\text { Campos }\end{array}$ & $\begin{array}{c}\text { Villamuriel de } \\
\text { Cerrato }\end{array}$ \\
\hline $\begin{array}{l}\text { Referencia implícita a los alumnos de } \\
\text { altas capacidades }\end{array}$ & Sí & Sí & Sí \\
$\begin{array}{l}\text { Referencia explícita a los alumnos de } \\
\text { altas capacidades }\end{array}$ & Sí & Sí & Sí \\
$\begin{array}{l}\text { Referencia explícita a otros alumnos } \\
\text { con necesidades educativas }\end{array}$ & Sí & Sí & 14 \\
$\begin{array}{l}\text { Frecuencia de contenidos implícitos } \\
\text { vinculados a las altas capacidades }\end{array}$ & 24 & 7 & 4 \\
$\begin{array}{l}\text { Frecuencia de contenidos explícitos } \\
\text { vinculados a las altas capacidades }\end{array}$ & 7 & 3 & 8 \\
$\begin{array}{l}\text { Frecuencia de contenidos explícitos } \\
\text { vinculados a otros alumnos con } \\
\text { necesidades educativas }\end{array}$ & 21 & 21 & \\
\hline
\end{tabular}

Fuente: Elaboración propia (2018) 
La aplicación ATLAS.TI permite obtener una visión complementaria al presentar de forma gráfica los memos que tienen su origen en la codificación de los datos obtenidos de las citaciones. Por ejemplo, la Figura 8 proporciona una visión general del memo referencias explícitas a los alumnos de altas capacidades. Puede verse que la frecuencia de contenidos es de 4 y cuáles son dichas referencias.

Figura 8 - Red de relaciones de referencias explícitas al alumnado de altas capacidades en el PAD de Villamuriel de Cerrato (2018)

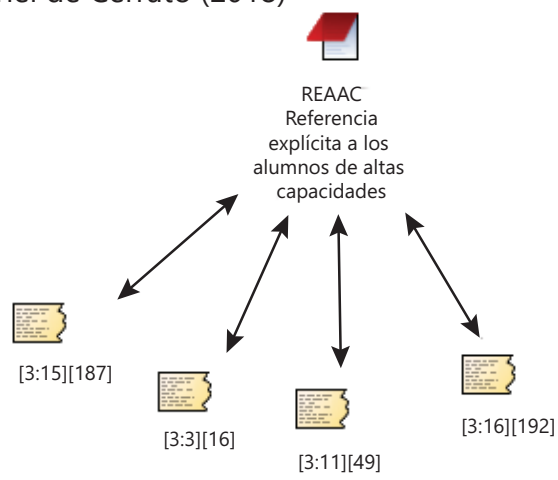

Fuente: Elaboración propia (2018)

En la Figura 9 se muestra la red de relaciones de referencias implícitas de otro de los PAD analizados.

Figura 9 - Red de relaciones de referencias implícitas al alumnado de altas capacidades en el plan de Becerril de Campos (2018)

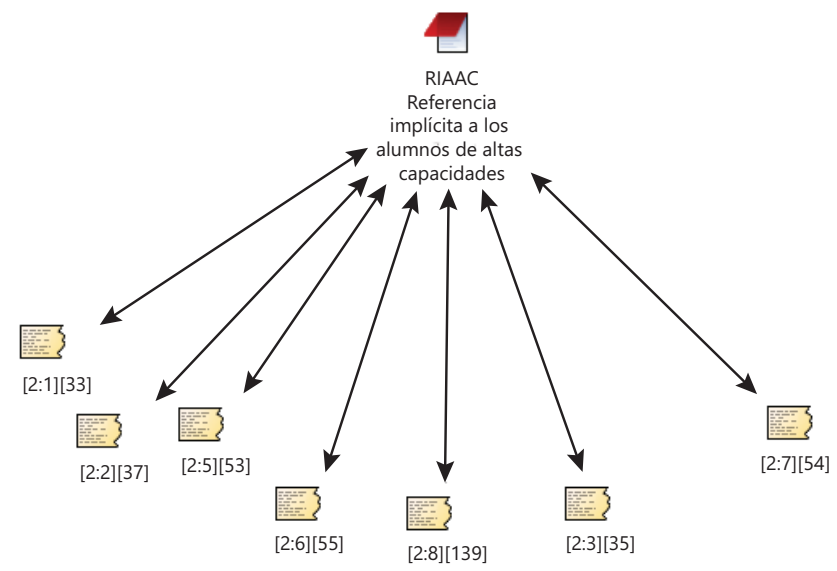

Fuente: Elaboración propia (2018) 
Por último, se muestra un ejemplo más de la red de relaciones de las referencias explícitas a otros grupos con necesidades educativas (Figura 10).

Figura 10 - Red de relaciones de referencias explícitas a otros grupos con necesidades educativas en el PAD de Villamuriel de Cerrato (2018)

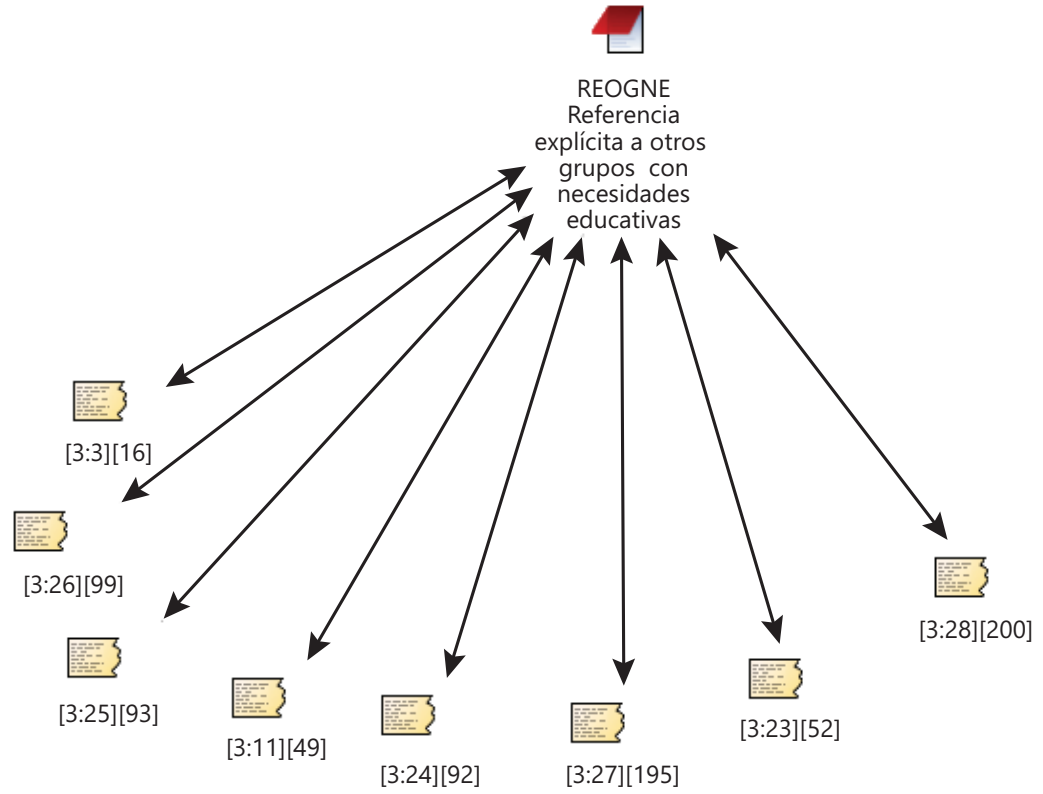

Fuente: Elaboración propia (2018)

\subsection{El proceso de categorización}

La categorización supone simplificar las valoraciones realizadas a las unidades de análisis de los PAD de la primera fase o las codificaciones de la segunda.

El dimensionamiento de ambos elementos puso de manifiesto una serie de elementos comunes que constituyen la base de la categorización realizada y que están conectados con las reglas de enumeración y los memos establecidos de antemano.

En la Figura 11 pueden verse los resultados del proceso de categorización. Hay que tener en cuenta que como dicho proceso es abierto, en la segunda fase se produjeron una serie de modificaciones que incidieron en las categorías obtenidas. 
Figura 11 - Proceso de reducción de las categorías de la primera y segunda fase (2018)

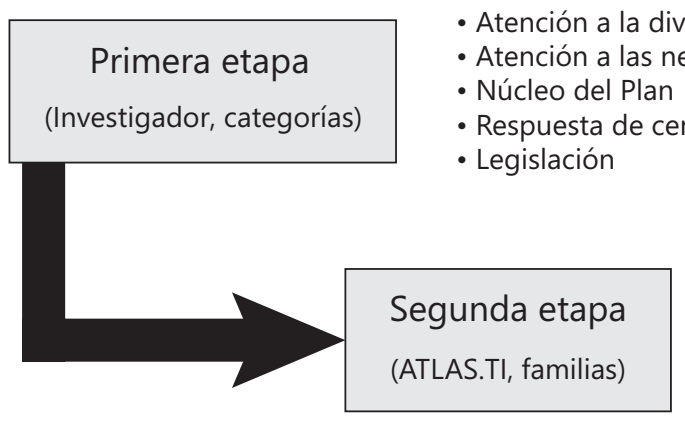

- Atención a la diversidad

- Atención educativa no ordinaria

- Atención educativa ordinaria

- Respuesta educativa inclusiva

Fuente: Elaboración propia (2018)

En ambas fases del proceso, el núcleo de la categorización son las referencias explícitas o implícitas a los alumnos más capaces o las diferencias con las referencias explícitas a la intervención con otros alumnos con necesidades educativas.

En la segunda fase la utilización de la aplicación informática ATLAS.TI permitió que se plasmasen de forma gráfica las relaciones existentes entre los memos y las familias. La Figura 12 muestra dichas relaciones.

La Tabla 4 muestra la relación entre los distintos componentes del proceso de categorización en la primera y la segunda fase.

Valoración de los resultados. Los resultados obtenidos en la primera fase de la investigación (Figuras 6 y 7) indican que sí aparecen contenidos explícitos vinculados a las altas capacidades. No obstante, se puede observar una baja frecuencia en la aparición de dichos contenidos. Por ejemplo, en el caso del PAD de la localidad de Astudillo, una de las referencias explícitas solo los menciona con relación a la tipología de alumnos con necesidades educativas especiales, es decir, alumnado de altas capacidades.

En la segunda fase igualmente aparecen contenidos explícitos vinculados a las altas capacidades y se produce un ligero incremento en la frecuencia de aparición en los planes, aunque sigue siendo baja. Tomados en conjunto, estos resultados sugieren una escasa consideración de los alumnos de altas capacidades en el proceso de Enseñanza y aprendizaje. 
Figura 12 - Memos y familias del análisis de contenido de los planes de la segunda fase (2018)

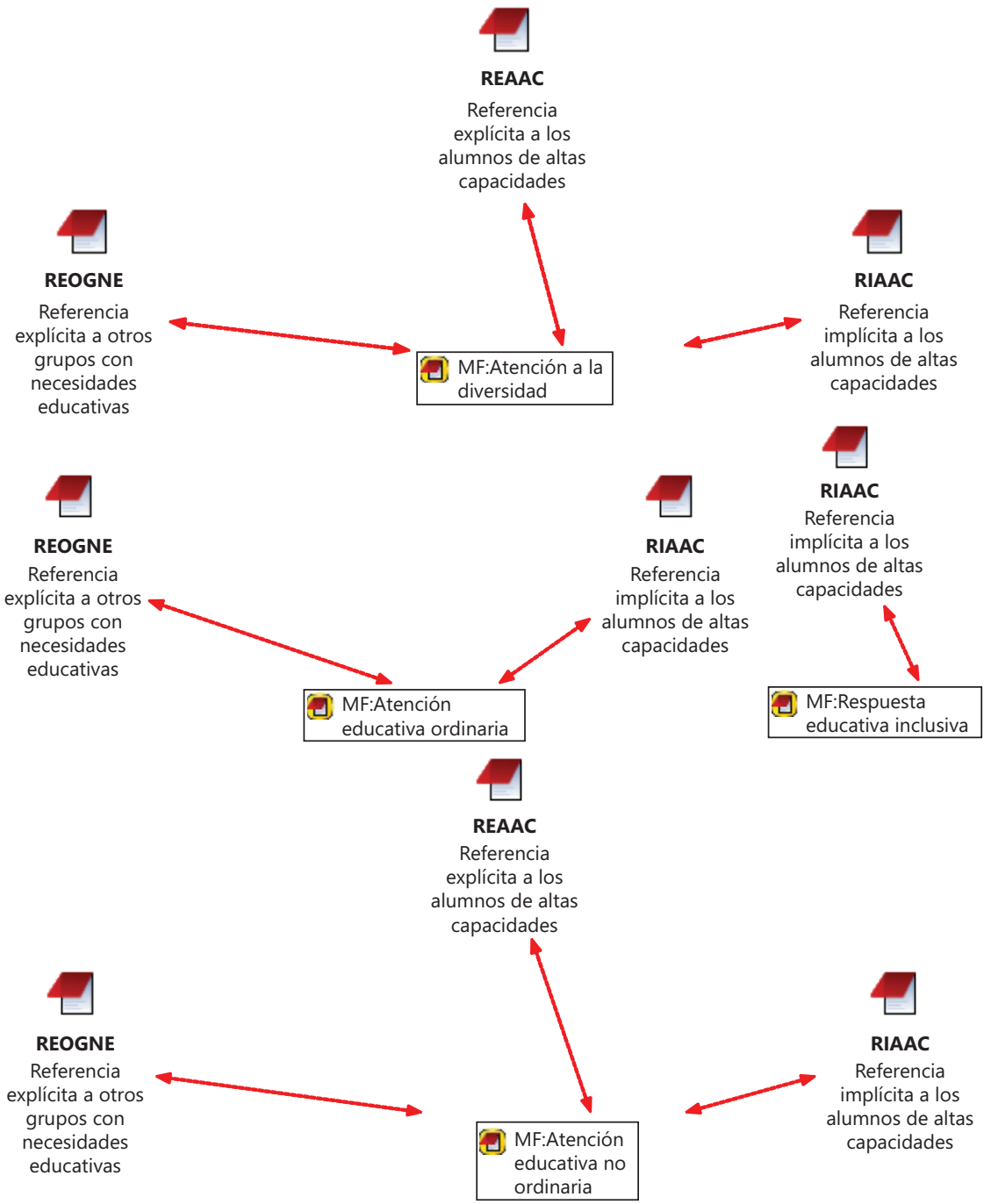

Fuente: Elaboración propia (2018)

En general, tanto las referencias implícitas como explícitas indican escasa incidencia en el desarrollo de los aspectos organizativos del proceso educativo, teniendo algo más de impacto en los ajustes que podrían realizarse a los elementos de la propuesta curricular. Por ejemplo, se mencionan aspectos tales como el 
Tabla 4 - Proceso de categorización seguido durante las dos fases del análisis de contenido (2018)

\begin{tabular}{|c|c|c|}
\hline & Reglas de enumeración & Categorías \\
\hline \multirow{5}{*}{ 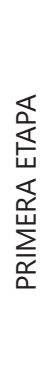 } & $\begin{array}{c}\text { Presencia /ausencia de contenidos implícitos/explícitos } \\
\text { vinculados a las altas capacidades }\end{array}$ & Atención a la diversidad \\
\hline & $\begin{array}{c}\text { Frecuencia de los contenidos explícitos/implícitos } \\
\text { vinculados a las altas capacidades o términos vinculados } \\
\text { a las mismas }\end{array}$ & $\begin{array}{c}\text { Atención a las necesidades } \\
\text { de los alumnos }\end{array}$ \\
\hline & $\begin{array}{l}\text { Frecuencia ponderada de los contenidos explícitos/ } \\
\text { implícitos vinculados a las mismas }\end{array}$ & Núcleo del plan \\
\hline & Orden o secuencia de aparición de los elementos & Respuesta de centro \\
\hline & explícitos/implícitos vinculados a las altas capacidades & Legislación \\
\hline \multirow{7}{*}{ 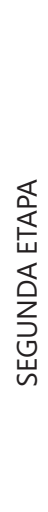 } & Memos & Familias \\
\hline & Referencia implícita a los alumnos de altas capacidades & Atención a la diversidad \\
\hline & Referencia explícita a los alumnos de altas capacidades & $\begin{array}{l}\text { Atención educativa no } \\
\text { ordinaria }\end{array}$ \\
\hline & $\begin{array}{c}\text { Referencia explícita a otros alumnos con necesidades } \\
\text { educativas }\end{array}$ & $\begin{array}{l}\text { Atención educativa } \\
\text { ordinaria }\end{array}$ \\
\hline & $\begin{array}{l}\text { Frecuencia de contenidos implícitos vinculados a las } \\
\text { altas capacidades }\end{array}$ & \\
\hline & $\begin{array}{l}\text { Frecuencia de contenidos explícitos vinculados a las altas } \\
\text { capacidades }\end{array}$ & $\begin{array}{l}\text { Respuesta educativa } \\
\text { inclusiva }\end{array}$ \\
\hline & $\begin{array}{l}\text { Frecuencia de contenidos explícitos vinculados a otros } \\
\text { alumnos con necesidades educativas }\end{array}$ & \\
\hline
\end{tabular}

Fuente: Elaboración propia (2018)

agrupamiento del alumnado y la coordinación y organización del profesorado, aunque no parece existir una relación directa con la Educación de los alumnos de altas capacidades.

Centrándonos en las referencias a contenidos explícitos, estas son prácticamente inexistentes o testimoniales en la primera fase (Ilustraciones 6 y 7), ocurriendo algo similar en los planes de la segunda, pues su repercusión en la práctica educativa no trasciende la normativa.

Los resultados relacionados con la existencia de contenidos implícitos son más numerosos e indican referencias potenciales para la Educación de los alumnos de altas capacidades que no necesariamente se van a plasmar en la práctica educativa. 
En este caso sí hay un avance respecto a los aspectos planteados en la legislación; sin embargo, dichos contenidos implícitos acabarán dirigidos hacia otros colectivos diversos identificados y visibles en la práctica educativa de los centros educativos analizados como son los alumnos con dificultades de aprendizaje. En este sentido, la calidad de la Educación sería mayor para dichos colectivos y la autonomía organizativa de los centros está más desarrollada para las dificultades de aprendizaje.

Una de las consecuencias del insuficiente desarrollo de propuestas de intervención para los más capaces es la inexistencia de un marco organizativo específicamente diseñado para su Educación.

Ello conlleva que ante la necesidad de intervenir con estos alumnos los elementos organizativos del centro estén diseñados y más preparados para otro tipo de necesidades educativas, y no sean adecuados para responder a las que se derivan de las altas capacidades.

Respecto al proceso de categorización, los agrupamientos de los contenidos de dichas unidades indican una escasa intervención con los alumnos más capaces, siendo mayores las posibilidades de intervención a través de referencias implícitas que son las que suponen el grueso de la categorización.

\section{Conclusiones y orientaciones para la práctica educativa}

La estructura organizativa es un componente básico para la Educación del alumnado en general y también para los alumnos de altas capacidades, al incidir en la forma en la que se llevan a la práctica las propuestas que se hacen a estos alumnos.

Algunos de los elementos constitutivos de dicha estructura, por ejemplo, el agrupamiento del alumnado, la organización del profesorado, los espacios y los tiempos, están presentes en distintas investigaciones relacionadas con la práctica educativa.

El tiempo dedicado a la tarea, la coordinación del profesorado, los procedimientos de agrupamiento del alumnado, la organización física del aula, son elementos que están presentes en las investigaciones que hacen referencia a las escuelas eficaces (MURILLO, 2008). 
El resultado del análisis de los PAD indica que las estructuras organizativas del sistema educativo en general, y de los centros de la muestra seleccionados, son poco flexibles y no propician el desarrollo de todo el potencial de los alumnos de altas capacidades.

Por tanto, es necesario realizar propuestas educativas específicas para los más capaces en el marco de una estructura organizativa más flexible e inclusiva. Estudios como el de Aiscow (2012) sobre escuelas inclusivas señalan algunas condiciones organizativas adecuadas para la atención a la diversidad. Entre ellas, el liderazgo compartido o la planificación conjunta por parte del profesorado pueden servir como indicadores que permitan enmarcar las propuestas que se hagan para los alumnos más capaces.

El liderazgo compartido proporciona a los directores de las escuelas un papel crucial para impulsar el diseño, puesta en práctica y valoración de planes educativos en colaboración con el profesorado y su posterior valoración, o a través de la consecución de recursos para la Educación de los más capaces.

Otro de los hallazgos de esta investigación es que los marcos de intervención plasmados en los PAD no van más allá de indicar la necesidad de atender a los más capaces, sin desarrollar propuestas concretas.

Es necesario avanzar en la construcción de un marco organizativo diseñado específicamente para los estudiantes con alta capacidad que permita una intervención más eficaz y que no solo incida en aspectos curriculares. Las nuevas tecnologías pueden ayudar en este cometido por su contribución a la individualización del proceso educativo, necesaria para dar respuesta a las peculiares características de aprendizaje de los alumnos de altas capacidades.

Las referencias para atender a la diversidad tienen un carácter general y se centran en el conjunto de la atención a la diversidad limitando la atención a los más capaces e invisibilizándoles respecto a la totalidad del alumnado.

Además, esta situación se produce en un contexto en el que la atención a la diversidad se centra básicamente en aquellos alumnos que presentan dificultades de aprendizaje y basa su intervención en proporcionar los recursos necesarios a estos estudiantes y no al resto, obstaculizando y limitando la Educación de los más capaces (GARCÍA, 2017). 
También cobra sentido la idea esbozada por García (2017) acerca del papel de los orientadores para contribuir a la supresión de las barreras que impidan la intervención con los más capaces. Una forma de implementar esta función sería mediante el asesoramiento para el diseño y desarrollo de los PAD con medidas educativas adecuadas a las necesidades que presentan dichos estudiantes.

En cuanto a las administraciones educativas, sería adecuado un mayor desarrollo de la normativa (COMES NOLLA et al., 2012) para poder llevar a la práctica una Educación que contemple, desde el punto de vista organizativo, medidas concretas y específicas de las que puedan beneficiarse los más capaces, que les hagan tener presencia entre los diferentes colectivos diversos.

Considerando que la autonomía de los centros es un instrumento al servicio de la calidad educativa, la respuesta educativa a los más capaces está pensada para otros colectivos y no originalmente para ellos.

Por un lado, sería necesario el desarrollo de dicha autonomía por parte de la administración estableciendo el marco y los términos en que debería producirse. Por otro lado, los centros deben verse asesorados y acompañados en su desarrollo haciendo hincapié en las variables organizativas que permitirían una Educación de calidad para los estudiantes de altas capacidades en la escolaridad obligatoria.

Por último, no parece que sea una prioridad de los centros intervenir con los más capaces. Sería la propia legislación en la que se basan los PAD la que induciría una presencia testimonial en las propuestas educativas realizadas para ellos.

El análisis de contenido ha evidenciado la existencia de estructuras organizativas diseñadas e implementadas para alumnos con dificultades de aprendizaje que normalizan, a la baja, al alumnado de altas capacidades. También ha puesto de manifiesto la necesidad de impulsar una práctica educativa con los alumnos más capaces que tenga un punto de partida con base en las características de su aprendizaje y las necesidades educativas que se derivan de ellas.

Para finalizar, la sociedad del conocimiento implica la necesidad de acelerar las mejoras en la Educación de los más capaces, y de los demás alumnos, para poder adquirir ventajas competitivas en la economía global y así poder formar un mayor número de innovadores y emprendedores que sean personas conscientes de su capacidad y del compromiso personal y social a asumir. 
En este sentido, es necesario que se desarrollen marcos organizativos relacionados directamente con las políticas educativas, pero sin perder de vista que la ejecución recae en los profesionales de las organizaciones educativas. De ahí la importancia de su formación y de tomar en consideración la percepción que tienen de la Educación de los más capaces, pues puede significar barreras o facilitadores de dicha Educación (HEUSER; WANG; SHAHID, 2017). 


\title{
A resposta educativa aos alunos mais capazes nos planos de atenção à diversidade
}

\section{Resumo}

A atenção à diversidade é um princípio básico do sistema educativo espanhol e a sua finalidade é dar resposta às necessidades dos alunos que o frequentam. Nesta diversidade, os alunos com altas habilidades vão ganhando certa visibilidade no diagnóstico e na intervenção. A normativa nacional estabeleceu o Plano de Atenção à Diversidade (PAD) como o instrumento básico para planejar, implementar e avaliar as medidas de atenção à diversidade dos diferentes grupos, entre eles, o dos alunos mais capazes. Pela técnica de análise de conteúdo, esta colaboração analisa os PAD numa amostra de estabelecimentos escolares da província de Palencia, na Comunidade Autónoma de Castela e Leão. Conclui que nos referidos planos, a presença de medidas educativas destinadas aos mais capazes é nula ou puramente testemunhal, não ultrapassando as regulamentações nas quais se fundamenta, pelo que se verifica ser necessário desenvolver esses planos na prática educativa real.

Palavras-chave: Altas habilidades. Identificação. Intervenção educativa. Plano de atenção à diversidade.

\section{The educational response to the most capable students in plans on attention to diversity}

\begin{abstract}
Attention to diversity is a core principle of the Spanish educational system. It aims at meeting the needs of the students enrolled in its institutions. This diversity has helped to give visibility to high intellectual potential students as per their diagnosis and intervention. National regulations lay down the Plan on Attention to Diversity (PAD, by its Spanish initials) as the essential tool to plan, implement and assess the measures concerning attention to the diversity of the different groups, inter alia, the most capable students. By using the content analysis technique, this collaboration analyzes PADs from a sample of schools in the Spanish province of Palencia, in the autonomous region of Castile-Leon. It concludes that in certain plans, the presence of educational measures aimed at the most capable students is zero or a mere token and does not go beyond the regulations on which they are based, thus it becomes necessary to develop these plans in actual educational practice.
\end{abstract}

Keywords: High intellectual potential. Identification. Educational intervention. Plan on attention to diversity. 


\section{Referencias}

AINSCOW, M. Haciendo que las escuelas sean más inclusivas: lecciones a partir del análisis de la investigación internacional. Revista Educación Inclusiva, Almería, v. 5, n. 1, p. 39-49, 2012.

CARDONA ANDÚJAR, J. Aprendizajes acumulativo y generativo de las organizaciones educativas. Revista Educación, San Jose, v. 32, n. 2, p. 73-84, 2008. https://doi.org/10.15517/revedu.v32i2.517

COMES NOLLA, G. et al. Análisis y valoración de la situación actual del alumnado con altas capacidades en España. Revista Educación Inclusiva, Almería, v. 5, n. 2, p. 129-40, 2012.

COMITÉ ECONÓMICO Y SOCIAL EUROPEO - CESE. Liberar el potencial de los niños y los jóvenes con gran capacidad intelectual. Bruselas, 2013. Disponible en: http://www.fundacionpryconsa.es/media/Pleno.pdf. Acceso en: 1 nov. 2018.

ESPAÑA. Decreto No 26/2016, 21 de julio de 2016. Por el que se establece el currículo y se regula la implantación, evaluación y desarrollo de la educación primaria en la comunidad de Castilla y León. Boletín Oficial de Castilla y León, 22 jul. 2016.

ESPAÑA. Ley orgánica No 2/2006, de 3 de mayo de 2006. De educación. Boletín Oficial del Estado, 4 mayo 2006.

ESPAÑA. Ley orgánica No 8/2013, de 9 de diciembre de 2013. Para la mejora de la calidad educativa. Boletín Oficial del Estado, 10 dic. 2013.

ESPAÑA. Ministerio de Educación, Cultura y Deporte. Estadísticas de la educación de la enseñanza no universitaria. Madrid, 2018. Disponible en: http://www.mecd.gob.es/servicios-al-ciudadano-mecd/estadisticas/educacion/ no-universitaria.html. Acceso en: 18 feb. 2018.

GAGNÉ, F. De los genes al talento: la perspectiva DMGT/CMTD. Revista de Educación, Madrid, n. 368, p. 12-39, abr./jun. 2015. https://doi.org/10.4438/1988-592X-RE-2015-368-289

GAIRÍN, J.; GOIKOETXEA, J. La investigación en organización escolar. Revista de Psicodidáctica, Bizkaia, v. 13, n. 2, p. 73-95, 2008.

GAIRÍN, J.; RODRÍGUEZ-GÓMEZ, D. Cambio y mejora en las organizaciones educativas. Educar, Barcelona, v. 47, n. 1, p. 31-50, 2011. 
GARCÍA, A. Las necesidades educativas especiales: un lastre conceptual para la inclusión educativa en España. Ensaio: Avaliação e Políticas Públicas em Educação, Rio de Janeiro, v. 25, n. 96, p. 721-42, jul./sep. 2017. https://doi.org/10.1590/s0104-40362017002500809

GARCÍA, J. L.; GONZÁLEZ, M. A.; BALLESTEROS, B. Introducción a la investigación en educación. Madrid: Universidad Nacional de Educación a Distancia, 2001.

HERNÁNDEZ TORRANO, D.; GUTIÉRREZ SÁNCHEZ, M. El estudio de la alta capacidad intelectual en España: análisis de la situación actual. Revista de Educación, Madrid, n. 364, pp. 251-272, abr./jun. 2014. https://doi.org/10.4438/1988-592X-RE-2014-364-261

HEUSER, B. L.; WANG, K.; SHAHID, S. Global dimensions of gifted and talented education: the influence of national perceptions on policies and practices. Global Education Review, New York, v. 4, n. 1, p. 4-21, 2017.

INSTITUTO NACIONAL DE TECNOLOGÍAS EDUCATIVAS Y DE FORMACIÓN DEL PROFESORADO - Intef. Resumen informe horizon 2014. Madrid: Ministério de Educación, Cultura y Deportes, 2014. Disponible en: http://educalab.es/documents/10180/38496/Resumen_Informe_Horizon_2014. pdf/d76b478b-0ccb-4a5d-af3d-021f25f34139. Acceso en: 1 nov. 2018.

INSTITUTO NACIONAL DE TECNOLOGÍAS EDUCATIVAS Y DE FORMACIÓN DEL PROFESORADO - Intef. Resumen informe horizon 2015. Madrid: Ministério de Educación, Cultura y Deportes, 2015. Disponible en: http://educalab.es/documents/10180/509115/Informe_Horizon_octubre_2015. pdf/6afb6039-41aa-4af0-93fb-b87ecab00c86. Acceso en: 1 nov. 2018.

INSTITUTO NACIONAL DE TECNOLOGÍAS EDUCATIVAS Y DE FORMACIÓN DEL PROFESORADO - Intef. Resumen informe horizon 2016. Madrid: Ministério de Educación, Cultura y Deportes, 2016. Disponible en: https://intef.es/wp-content/uploads/2016/10/2016_1018_Resumen_ Horizon_Primaria_Secundaria_2016_INTEF.pdf. Acceso en: 1 nov. 2018.

INSTITUTO NACIONAL DE TECNOLOGÍAS EDUCATIVAS Y DE FORMACIÓN DEL PROFESORADO - Intef. Resumen informe de tendencias kennisnet: cómo las TIC preparar a los estudiantes para el futuro. Madrid: Ministério de Educación, Cultura y Deportes, 2017. Disponible en: https:// intef.es/wp-content/uploads/2017/02/2017_0221_Tendencias_TIC_Kennisnet_ INTEF.pdf. Acceso en: 1 nov. 2018. 
JIMÉNEZ, C. et al. Hacia un modelo sociocultural explicativo del alto rendimiento y la alta capacidad: ámbito académico y capacidades personales. Educación XX1, Madrid, v. 13, n. 1, p. 125-53, 2010.

MURILLO, F. J. Hacia un modelo de eficacia escolar: estudio multinivel sobre los factores de eficacia en las escuelas españolas. Reice: Revista Electrónica Iberoamericana sobre Calidad, Eficacia y Cambio en Educación, Madrid, v. 6, n. 1, p. 4-28, 2008.

OAKES, J. Keeping track: how schools structure inequality. New Haven, CT: Yale University, 1985

RENZULLI, J. The three-ring conception of giftedness: a developmental model for promoting creative productivity. In: STERNBERG, R. J.; DAVIDSON, J. (eds.). Conceptions of giftedness. Boston: Cambridge University, 2005. p. 217-45. Disponible en: https://www.researchgate.net/ publication/237668711_The_Three-Ring_Conception_of_Giftedness_A_ Developmental_Model_For_Promoting_Creative_Productivity. Acceso en: 9 nov. 2018.

RENZULLI, J. S.; GAESSER, A. H. Un sistema multicriterial para la identificación del alumnado de alto rendimiento y de alta capacidad creativoproductiva. Revista de Educación, Madrid, n. 368, p. 96-131, abr./jun. 2015. https://doi.org/10.4438/1988-592X-RE-2015-368-290

ROMÁN GONZÁLEZ, M. Aprender a programar "apps” como enriquecimiento curricular en alumnado de alta capacidad. Bordón: Revista de Pedagogía, Madrid, v. 66, n. 4, p. 135-55, 2014. https://doi.org/10.13042/Bordon.2014.66401

STEENBERGEN-HU, S.; MAKEL, M. C.; OLSZEWSKI-KUBILIUS, $\mathrm{P}$. What one hundred years of research says about the effects of ability grouping and acceleration on $\mathrm{K}-12$ students' academic achievement: findings of two second-order meta-analyses. Review of Educational Research, London, v. 86, n. 4, p. 849-99, dic. 2016. https://doi.org/10.3102/0034654316675417

TOURÓN, J.; SANTIAGO, R. El modelo flipped learning y el desarrollo del talento en la escuela. Revista de Educación, Madrid, n. 368, p. 196-231, abr./ jun. 2015. https://doi.org/10.4438/1988-592X-RE-2015-368-288

TOURÓN, J.; TOURÓN, M. La enseñanza a distancia: posibilidades para la atención individualizada de los alumnos de alta capacidad en la escuela y la 
familia. Revista Española de Pedagogía, Madrid, v. 66, n. 240, p. 297-314, mayo/ago. 2008.

WALKER, C. L.; SHORE, B. M.; FRENCH, L. R. A theoretical context for examining students' preference across ability levels for learning alone or in groups. High Ability Studies, London, v. 22, n. 1, p. 119-41, jul. 2011. https://doi.org/10.1080/13598139.2011.576082

WINEBRENNER, S.; BRULLES, D. The cluster grouping handbook: a schoolwidemodel: how to challege gifted and improve achivement for all. Minneapolis: Free Spirit, 2008.

\section{Información del autores}

Lorenzo Pérez Diez: Doctor en Educación. Escuela Internacional de Doctorado de la Universidad Nacional de Educación a Distancia, España. Contato: jos_eph@hotmail.com (iD) http://orcid.org/0000-0001-8542-108X

Carmen Jiménez Fernández: Doctora en Educación. Catedrática Emérita. Facultad de Educación de la Universidad Nacional de Educación a Distancia, España. Contato: mjimenez@edu.uned.es

iD http://orcid.org/0000-0002-5899-623X 